\title{
PREVALENCE OF SCHISTOSOMA HAEMATOBIUM INFECTION IN GHANA: A RETROSPECTIVE CASE STUDY IN KUMASI
}

\author{
SAMMY CROWTHER KOFI TAY ${ }^{1}$, RICHCANE AMANKWA ${ }^{1}$ AND STEPHEN YAO GBEDEMA ${ }^{2^{\star}}$ \\ 1Department of Clinical Microbiology, School of Medical Sciences, College of Health Sciences, KNUST, Kumasi, Ghana \\ 2Department of Pharmaceutics, Faculty of Pharmacy and Pharmaceutical Sciences, College of Health Sciences, KNUST, \\ Kumasi, Ghana \\ *Corresponding Author: Email- sgbedema.pharm@knust.edu.gh; Tel. +233243301676
}

Received: September 09, 2011; Accepted: November 25, 2011

\begin{abstract}
In Ghana, urinary schistosomiasis was considered a public health problem in the rural communities only. Recent reports are pointing to the resurgence of this infection in urban and peri-urban settlements. In this study, the prevalence of Schistosoma haematobium infection presenting at Kumasi South Hospital (surrounded by Atonsu, Gyinyaase and Ahinsan communities) and Aninwa Medical Centre (surrounded by Boadi, Ayeduase, Emena, Appiadu and Kokoben) were investigated in retrospect to determine whether $S$. haematobium infection rate difference was consistent over time and also to compare the prevalent rates of the infection between these two community hospitals. The available records of patients referred for urinalysis between 2000 and 2009 were retrospectively reviewed. Prevalence of $S$. haematobium infection at Kumasi South Hospital (an urban poor community) was 40.2\% whilst Aninwa Medical Centre (a peri-urban settlement) recorded $4.5 \%$. The infection was higher among males (66.4\%) than the females (33.6 \%) at both hospitals and a general pattern of 3- year peaks of the infection was observed. Our findings have indicated that the urban poor communities around Kumasi South Hospital are seriously affected by S. haematobium infection as compared to Aninwaah Medical Centre. Economic, agricultural and recreational activities of these communities living around the banks of River Susan in addition to lack of good household water might account for the high risk of the infection in the urban poor communities in the catchment areas of the Kumasi South Hospital.
\end{abstract}

Key Words- urban-poor, schistosomiasis, haematuria, Bulinus species

\section{Introduction}

Schistosomiasis, one of WHO's "most neglected diseases" is a major tropical and subtropical disease commonly found widespread in many African countries and other developing countries in Asia and South America. It is the second most prevalent tropical disease after malaria [1]. Climate changes are found to have effect on the transmission of the helminth parasites which have intermediate hosts in their life cycles. The WHO estimated that over 150,000 deaths and a burden of 5.5 million disability-adjusted life years can be attributed to climate change and variability each year [2]. Climate warming is accompanied by perturbations in the global hydrologic cycle and pronounced changes in water availability which then affects diseases spread by water including urinary schistosomiasis because changes in rainfall have an impact on the flow of rivers and levels of lakes [3]. These predictive profiles of climate-sensitive diseases are collectively worsening for most parts of the world [2].

Over 200 million people are infected with Schistosoma haematobium world-wide [1]. There are reports of high prevalence of the infection particularly among children who acquire the infection by being exposed to fresh water containing the infective cercariae of the $S$. haematobium $[4,5]$. Urinary schistosomiasis is a chronic disease usually characterised by haematuria [4]. This parasitic infection imposes significant economic burdens on individuals, communities and nations [6]. A survey in the year 2000 of the disease-specific mortality reported that 70 million individuals, out of 682 million, had experienced haematuria and 32 million, dysuria associated with S. haematobium infection [4]. The WHO estimates that out of these infected individual 18 million suffered cancer of the bladder and 10 million hydronephrosis [7]. In addition, estimates of about 280,000 deaths relating to schistosomiasis occur annually in sub-Saharan Africa [7]. The disease therefore, constitutes an important public health problem in this region [8]. In Ghana, the prevalence of this infection ranges between $54.8 \%$ and $60.0 \%$ and this is an indication that the disease is still a major problem in some parts of the country [9].

In this study two health facilities in the Kumasi Metropolis were retrospectively assessed for the prevalence of $S$. haematobium infection to determine the extent of public health education about the disease in the urban setting. This will provide useful data for combating the infection in this part of the country.

Material and Method Ethical Issues 
The study protocol was approved by the Committee on Human Research, Publications and Ethics, School of Medical Sciences, Kwame Nkrumah University of Science and Technology in collaboration with the managements of Kumasi South Hospital and Aninwaah Medical Centre which also granted the permission to undertake the study. Information obtained from both hospitals were kept confidential and used only for this research.

\section{Study design}

The study was conducted by reviewing a ten year period (from January 2000 to December 2009) medical records of patients who reported at the Kumasi South Hospital (Atonsu Hospital) and Aninwaah Medical Centre (Emena Hospital) laboratories in the Kumasi Metropolis (Fig 1) for urinalysis.

\section{Review of medical records}

Data of number of patients with haematuria, number of patients who were positive for $S$. haematobium ova and sex of the patients within the period of study were obtained. Parasitic infection of $\mathrm{S}$. haematobium was considered positive when the egg of $\mathrm{S}$. haematobium was identified on examination of urine sediments with the aid of a light microscope.

\section{Examination of urine}

Urine centrifugation concentration conventional technique was used. Ten milliliters of urine samples after centrifugation at RCF $500-1000 \mathrm{~g}$ for 5 minutes each was examined microscopically for the Schistosoma haematobium eggs [5].

Data analysis

Microsoft Excel 2007 was used to analyze the data collected from the two hospitals.

\section{Results}

\section{Baseline characteristics of the study}

Out of a total of 1569 patients with symptoms of haematuria at the Kumasi South Hospital (709) and Aninwah Medical Centre (860) between January 2000 and December 2009 examined, S. haematobium ova were detected in the urine samples of $20.7 \%$ (324) whilst $79.3 \%$ (1245) did not have the ova. The overall prevalence of the infection at Kumasi South Hospital (surrounded by urban-poor communities) was about nine times higher $(40.2 \%)$ than that of Aninwa Medical Centre $(4.5 \%)$ in a peri-urban settlement. The highest cases of the infection (65\%) were recorded in 2008 at the Kumasi South Hospital while Animwa Medical Centre recorded its highest cases (12.61\%) in 2007. While Kumasi South Hospital recorded erratic episodes of the infection in some of the years with total absence in others, the infection was low and persistent in the Animwa Medical Centre throughout the study period (Fig 2).

Distribution of $S$. haematobium infections between males and females patients

Male patients constituted a higher proportion (66.4\%) of the overall 324 cases of the $S$. haematobium infection recorded in the two hospitals (Fig 3). The number of males reporting the infection was persistently higher than their female counterparts throughout the period of study. No female patients were diagnosed of the infection in 2002, 2003 and 2006.

The highest prevalence occured in January (15.4\%) and the minimum in March (3.4\%). The recorded prevalences were inconsistent between April and December, and were in the range of $5.86 \%$ and $10.19 \%$ (Fig 4).

Yearly trend of S. haematobium infection in the two hospitals

The S. haematobium infection was highest (32.72\%) in 2000. Relatively high cases were again recorded in 2001, 2004 and 2008; the number of cases was however slightly lower (8.95 \%) in 2009 (Fig 5).

\section{Limitations}

The ages of the patients were not available in the records; hence the most susceptible age group could not be determined. There were no available records for some of the years; hence prevalence for those years could not be determined.

\section{Discussion}

Urinary schistosomiasis is of much public health concern especially in developing countries. It imposes extensive economic and health implications on residents living in endemic areas. The prevalence of $S$. haematobium infection recorded during the period of ten years (2000 to 2009 ) in this study was $20.7 \%$. However, prevalence rates reported at Aninwaah Medical Centre and Kumasi South Hospital were $4.5 \%$ and $40.2 \%$ respectively. The difference in the rates could be due to differences in the number of freshwater bodies infested with the snail intermediate host (Bulinus species) of the parasite as well as the behavioural patterns of the communities.

Various factors could be associated to the high rate of S. haematobium infection recorded in the Kumasi South Hospital. These include unsanitary conditions, unavailability of clean household water and some human recreational and behavioural practices such as swimming, fishing, fetching and washing in water infested with the snail intermediate hosts of the parasite [11-13]. Atonsu township where Kumasi South Hospital is located (Fig 1), exhibits characteristic of urban poor settlement and has pockets of rivers including Susan and Wewe rivers and these are used for recreation, farming, washing and fishing activities. These freshwater bodies are known to be infested with the freshwater snails. Animwa Medical Centre, located in Emena, however recorded much lower cases of the infection. This is because Emena township exhibits characteristics of periurban settlement; it is a fairly new developing area with most of the residents having wells and pipe borne water in their homes. However, very few residents who lack pipe borne water rely on pockets of ponds and the few streams in the community for household water. The presence of good household water may have contributed to the differences in the prevalence rates of schistosomiasis recorded in the two hospitals. 
The result of this study is consistent with reports by Yeboah [14] and Clennon et al. [15] suggesting that proximity of household to infected water bodies is significant in the transmission of $S$. haematobium infection. Increase contact time of the infested water bodies by people around Kumasi South Hospital might also have accounted for the high prevalence of the infections in these communities compared with the communities around Aninwaah Medical Centre $[16,17]$. A similar study conducted by Aboagye and Edoh [10], in the Ga District also revealed high prevalence rates of the infection in Mahem (58\%) which is closer to the Weija dam than Galilea (49\%) community. Our study is also consistent with those reported by Okolie and Odaibo [18] from Ibadan in Nigeria which showed a prevalence rate of $17.4 \%$. Urinary schistosomiasis is therefore a major public health problem in the sub-region; not only in the rural areas but also in both urban and peri-urban communities [19].

Higher prevalence of the infection was recorded among males than females. This is probably due to increase contact with infected water bodies by males than female as result of engagement in swimming and or agriculture activities. Nsowah-Nuamah et al. [9] also reported high rates of the infection among males (55.9\%) than the female counterparts (3.7\%). Chimbari and Chirundu [20] conducted a similar study in Zimbabwe which also revealed higher infection rates among males than the females. These observations are expected considering the fact that some socio-cultural practices such as farming, fishing and recreational activities expose males to infected water bodies than the female counterparts [21,22].

The major rainy seasons in Ashanti Region run from April to June with the infection prevalence ranging between $6.8 \%$ and $9.0 \%$. The minor rainy seasons also runs from November to February with prevalence ranging from $5.9 \%$ to $15.4 \%$. Rainfall is associated with the distribution of snail species [23]. The abundant aquatic weed that develops during the raining seasons serves as readily available food for the snails. The Bulinus species therefore reproduce extensively in the raining season and by the beginning of the dry season where there is increased human activity with these fresh water bodies, these freshwater snails become infected with the miracidium which develop into the infective cercariae and upon release penetrate the skin of individuals who come into contact with the infested water [23]. In our study, this appears to account for the increase in the infections in the dry and minor rainy seasons as compared to the major rainy season.

There was yearly trend of $S$. haematobium infection during the period under review. This has shown that after every three years, prevalence of $S$. haematobium infection increases with peaks values of $32.7 \%, 17.0 \%$ and 14.5 as observed in 2000, 2004 and 2008 respectively. This has also shown that the prevalence rates in these years were declining over time. This present study has demonstrated that $S$. haematobium in these study sites showed a three-year seasonal pattern of major increase with time. This may be due to increase in survival of the snail intermediate host in favourable environment after every three years [17]. Thus, results obtained from both hospitals in this present study shows that the prevalence of $S$. haematobium infection is seasonal.

Control programmes based on oral drug delivery have been developed and particularly implemented as a means to control morbidity in endemic areas [24]. Water sanitation, mass chemotherapy, of infected people with drugs such as praziquantel and health education to highlight the disease, are some of the preventive measures [25].

\section{Conclusion}

The prevalence of $S$. haematobium infection over the past ten years was $20.7 \%$ at both health facilities with Kumasi South Hospital recording higher prevalence than Aninwaah Medical Centre. S. haematobium infection was predominant among males patients than in females. There was a general three-year pattern of $S$. haematobium peaks of infection during the period under review with infection occurring throughout the year, hence the need for intervention. Education of the various communities must be encouraged in addition to the provision of adequate household water, private and public toilets and communal laundry.

There is a need for future prospective studies of residents in these communities. Result further suggests the design of programmes for control of schistosomiasis in community study sites based on prophylactic treatment during the dry season to control the morbidity associated with infection.

\section{Acknowledgement}

The Arthurs will like to acknowledge the Government of Ghana for providing funds for this study.

\section{References}

[1] Lengeler C., Utzinger J. and Tanner M. (2002) Bull World Health Organ, 80(3): 235-242.

[2] Zhou X.N., Yang G.J., Yang K, Wang X.H., Hong Q.B., Sun L.P., Malone J.B., Kristensen T.K., Bergquist N.R. and Utzinger J. (2008) American Journal of Tropical Medicine and Hygiene, 78(2):188-194.

[3] Shope R.E. (1992) Global Climate Change: Implications, challenges and mitigation measures, Ed. Majumdar, S.K., Kalkstein, L.S., Yarnal, B., Miller, E.W., Rosenfeld, L.M. and Easton, P.A. The Pennsylvania Academy of Science, 1992: 363-370.

[4] van der Werf M.J., de Vlas S.J., Brooker S., Looman C.W.N.,Nagelkerke N.J.D., Habbenma J.D.F., Engels D. (2003) Acta Tropica, 86(2-3): 125-139.

[5] Cheesebrough M. (2005) District laboratory practice in tropical countries. 2 edn. Cambridge University Press: New York, 236-239. 
[6] Blas B.L., Lipayon I.L., Tormis, L.C., Portillo L.A., Hayashi M. and Matsuda H. (2006) Southeast Asian Journal of Tropical Medicine and Public Health. 37(1): 26-32.

[7] King C.H., Dickman K. and Tisch D.J. (2005). Lancet, 365(9470): 1561-1569.

[8] Taylor M. (2008) Bull World Health Organ, 86(10): 738.

[9] Nsowah-Nuamah N.N., Mensah G., Aryeetey M.E., Wagatsuma Y. and Bentil G. (2001) American Journal of Tropical Medicine and Hygiene, 65(5): 484-490.

[10] Aboagye I.F. and Edoh D. (2009) West African Journal of Applied Ecology, 15: 4.

[11] Wagatsuma Y., Aryeetey M.E., Nkrumah F.K., Sack D.A. and Kojima S. (2003) Central African Journal of Medicine, 49(1-2): 16-9.

[12] Useh M.F. and Ejezie G.C. (1999) Annal of Tropical Medicine \& Parasitology, 93(7): 711720.

[13] Zakhary K. (1997) McGill Journal of Medicine, 3: 93-101.

[14] Yeboah S.K. (1981) The problem of Bilharziasis transmission on River Boku, Kumasi, MSc. Thesis, KNUST Printing Press, Kumasi, Ghana.1-77.

[15] Clennon J.A., King C.H., Muchiri E.M., Kariuki H.C., Ouma J.H., Mungai P. and Kitron U. (2004) American Journal of Tropical Medicine and Hygiene, 70: 443-448.

[16] Mbata T., Orji M. and Oguoma V. (2008) The Internet Journal of Epidemiology, 6 (1).
[17] Kloos H., Fulford A.C.A., Butterworth A.E., Sturrock R.F., Ouma J.M., Kariuki H.C., Thiongo F.W., Dalton P.R. and Klumpp R.K. (1997) Social Science \& Medicine, 44: 949968.

[18] Okolie I. and Odaibo A.B. (1999) Tropical Medicine \& International Health, 14(4): 308-315.

[19] Agnew-Blais J., Carnevale J., Gropper A., Shilika E., Bail R. and Ngoma M. (2010) Journal of Tropical Paediatrics, 56(4): 247 253.

[20] Chimbari M.J. and Chirundu D. (2003) African Journal of Medicine, 49(1-2):8-12.

[21] Nmorsi O.P.G., Egwunyenga O.A. and Okholo O.E. (2001) South-East Asian Journal of Tropical Medicine \& Public Health, 3(3): 570574.

[22] Yapi Y.G., Briet O.J., Diabate S., Vounatsou P., Akodo E., Tanner M. and Teuscher T. (2005) Acta Tropica 93(2): 201-211.

[23] Kariuki H.C., Clennon J.A., Brady M.S., Kitron U., Sturrock R.F., Hoffman O., Hamburger J., Ouma J., Tosha S., Ndzovu M., Munga P., Pelligreni C., Muchiri E. and King C.H. (2004) American Journal of Tropical Medicine and Hygiene, 70(4): 443-456.

[24] WHO (2002) World Health Organ Technical Report Series, 912: i-vi, 1-57.

[25] Elderdery A., Eltoum M., Babiker A. and Hani B. (2008) Urological Cancer, 3(4): 18-19.

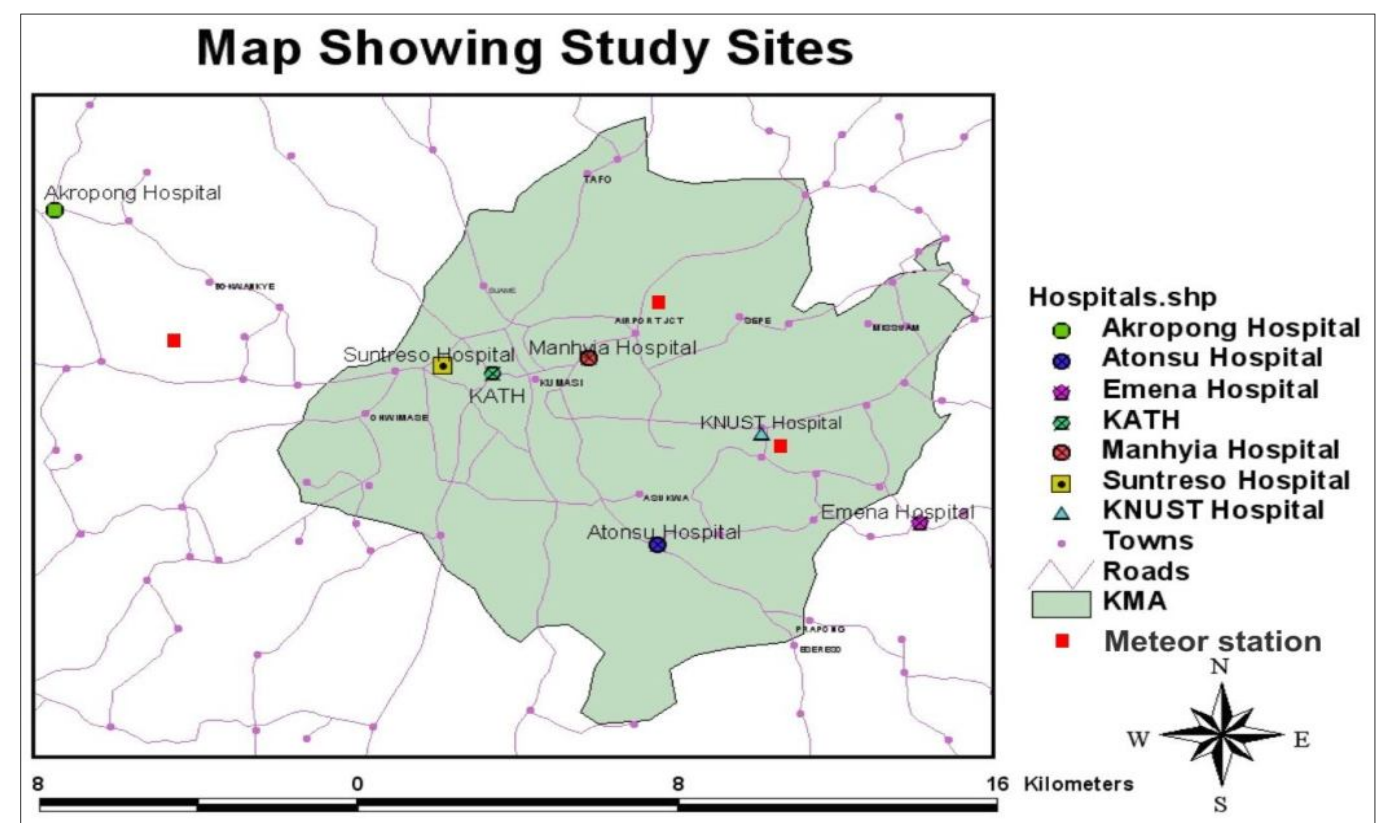

Fig 1- Map showing the study sites 


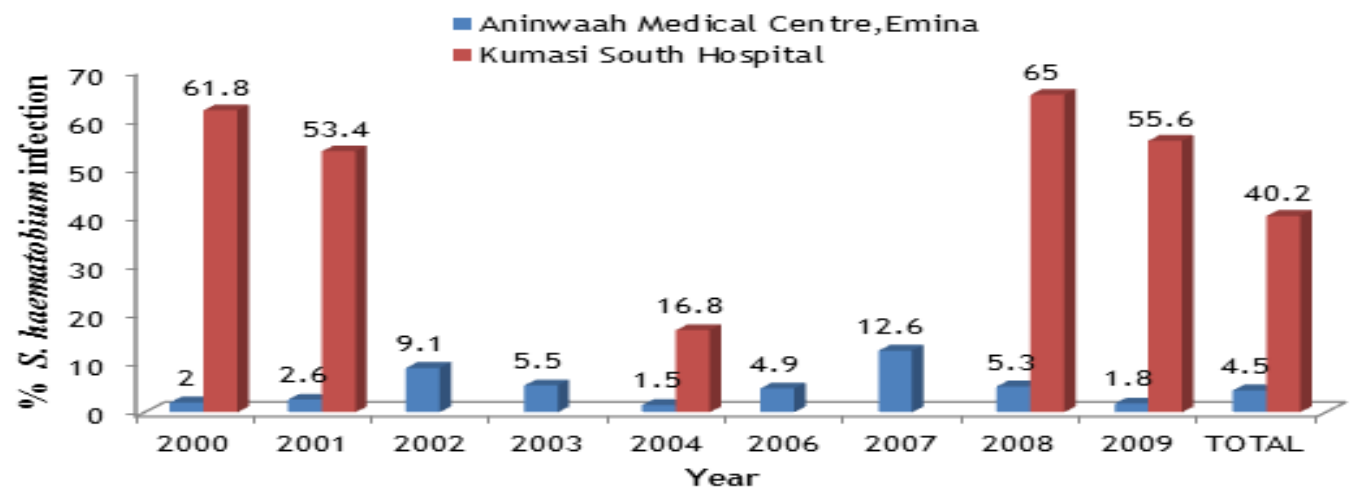

Fig 2- Prevalence of $S$. haematobium infection reported in two hospitals in Kumasi.

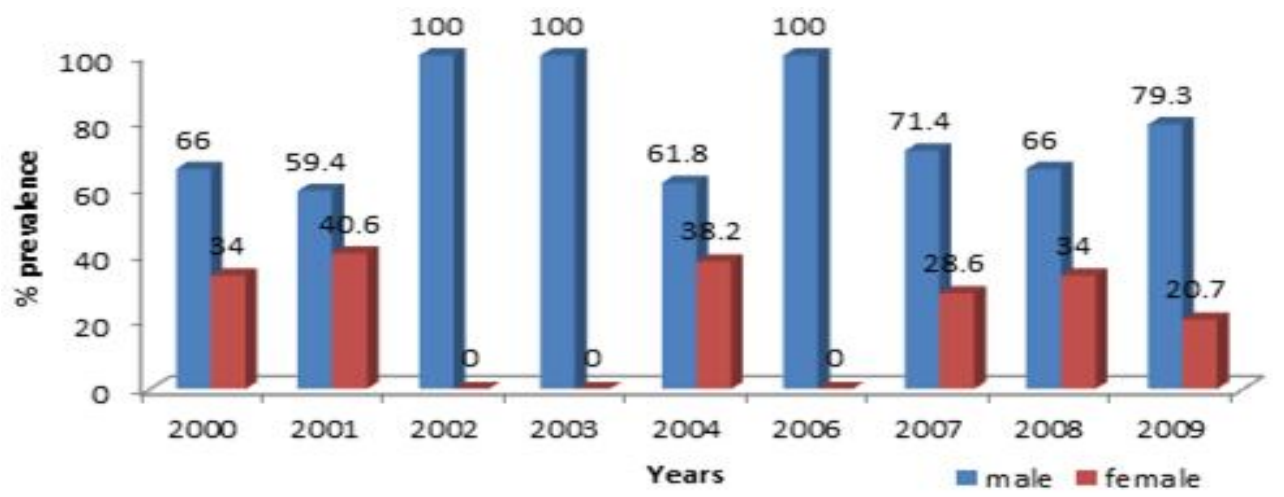

Fig 3- Distribution of $S$. haematobium infections among male and female patients

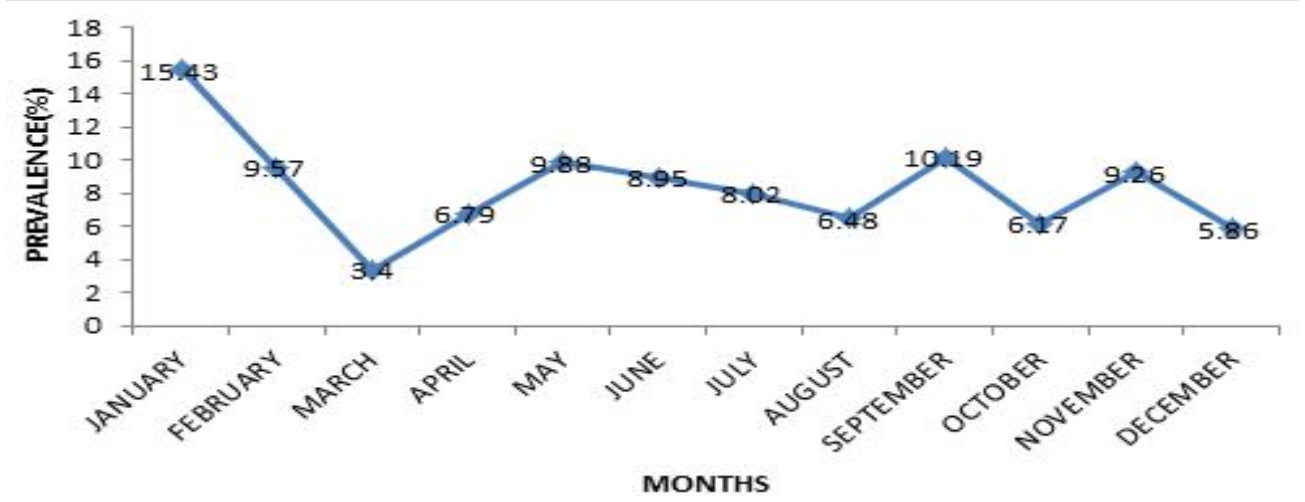

Fig 4- Total monthly distribution of $S$. haematobium infection in the two hospitals

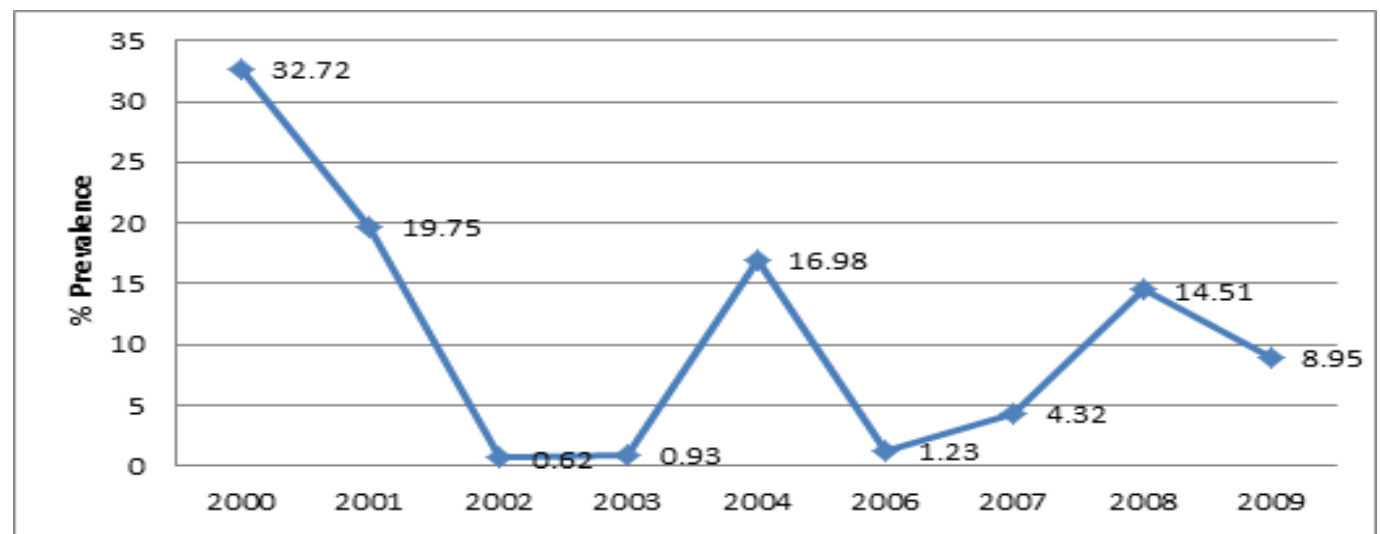

Fig 5- Total yearly distribution of $S$. haematobium infection in the 2 hospitals. 